\title{
Optogenetics: illuminating the role of PV interneuron dysfunction in the cognitive symptoms of schizophrenia
}

\author{
Madeline E. Parker*
}

\begin{abstract}
Our understanding of the neural substrates underlying the cognitive symptoms of schizophrenia remains limited, contributing to a lack of pharmaceuticals effective in treating these deficits. Cognitive impairment is the main source of disability in schizophrenia. Thus, research endeavours involving the identification of therapeutic targets can vastly improve the lives of affected individuals. Compared to previously employed techniques, optogenetics has accelerated such endeavours by allowing for manipulations of neuronal activity to occur with an unprecedented combination of cellular and temporal specificity. Research using this technique has united multiple, once tangentially-related, streams of evidence suggesting dysfunctional fast-spiking parvalbumin (PV) containing interneurons are a common substrate underlying the cognitive aberrations documented in schizophrenia. This review summarizes relevant optogenetic studies and identifies areas for future research, using this emerging technique.
\end{abstract}

Keywords: Optogenetics, schizophrenia, PV-interneuron, cognitive impairment

\section{Introduction}

Schizophrenia is an essentially incurable neuropsychiatric illness (Huang et al. 2013), affecting approximately $1 \%$ of the global population (Lewis et al. 2000). Historically, it was described in terms of positive symptoms, such as hallucinations and delusions, and negative symptoms, such as anhedonia and flattened affect (Addington et al. 1991; Cho et al. 2014). Cognitive symptoms are a more recently recognized core aspect of this disorder and include impairments in attention, memory, processing speed, social cognition, problem-solving, and perception (Geyer and Gross 2012). These deficits are present in nearly all individuals with schizophrenia and are the primary source of disabilities in social and economic functioning (Geyer and Gross 2012). Despite this, currently available antipsychotics only modestly improve cognition (Levkovitz et al. 2010) as our incomplete understanding of the underlying neural bases has hindered psychopharmaceutical development (Cho et al. 2014). Progress in this area will likely be informed

* Department of Anatomy, Physiology and Pharmacology, College of Medicine, University of Saskatchewan, Saskatoon, SK, Canada Correspondence: madeline.parker@usask.ca

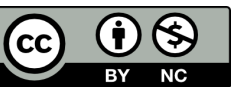

University of Saskatchewan Undergraduate Research Journal Volume 6, Issue 2, 2020

(c) 2020 Madeline E. Parker. This open access article is distributed under a Creative Commons Attribution Non-commercial 4.0 license. (https://creativecommons.org/licenses/by-nc/4.0/) 

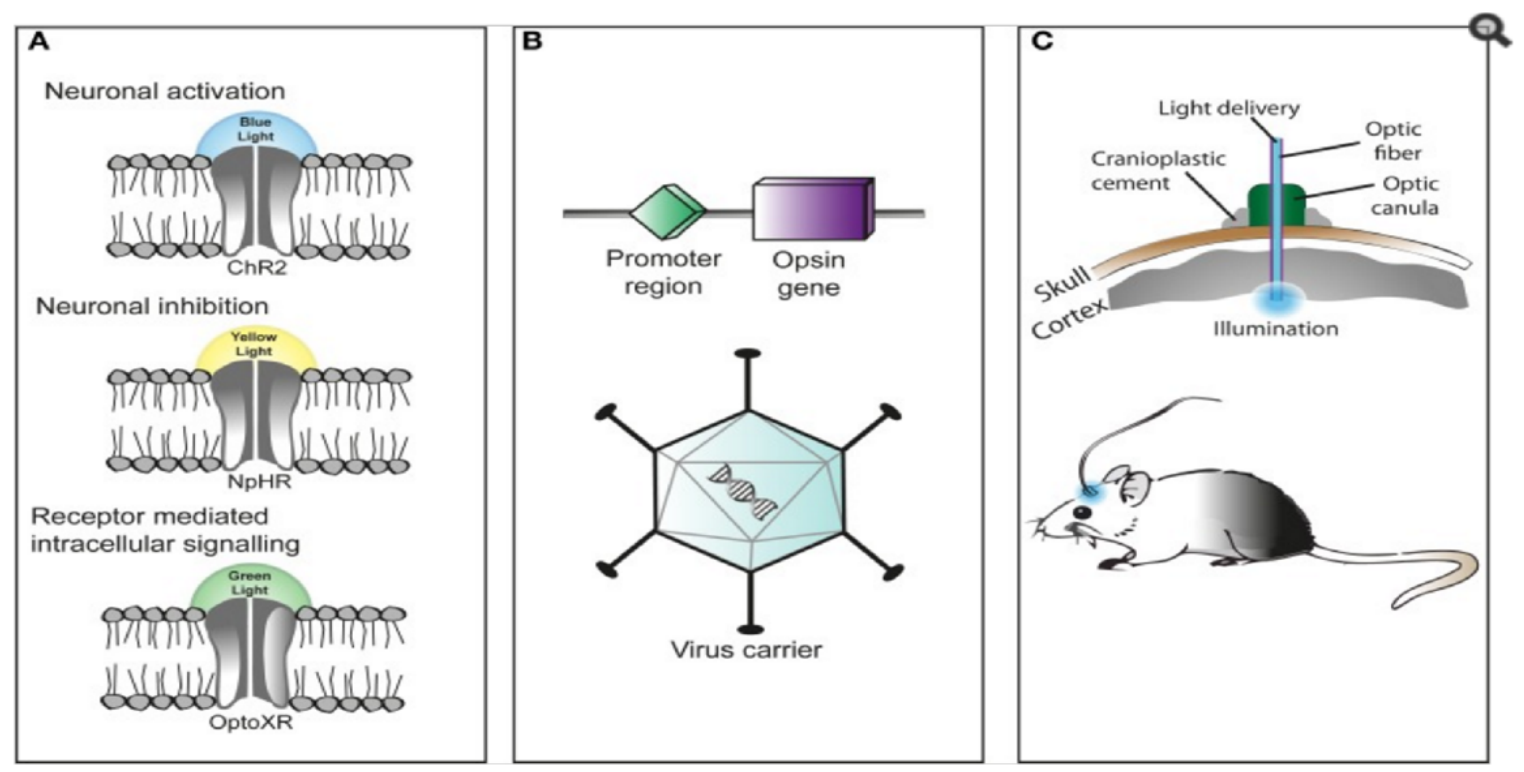

Figure 1: Optogenetic systems include: (A) opsins which confer activation, inhibition, or alterations in cellular signaling, (B) a viral delivery system containing the opsin's gene and corresponding promoter, and (C) light delivery via optic fibers implanted into the area of interest via canula. (Claudia et al., 2013)

by a growing body of evidence suggesting that disturbances in fast-spiking, parvalbumin-containing (PV), GABAergic interneurons underlie the cognitive symptoms of schizophrenia. Nevertheless, previous biochemical, genetic, electrophysiological, and pharmacological techniques have failed to reveal a direct link between PV interneurons and cognitive impairment. In contrast, due to its unparalleled combination of temporal and cellular specificity, optogenetics has significantly advanced our understanding of the cognitive aberrations associated with schizophrenia. Unlike previous approaches, this technique has provided the first causal evidence demonstrating that dysfunctional PV interneurons are the cellular correlates of these impairments.

\section{Optogenetic tools for Schizophrenia Research}

Optogenetics modulates neuronal activity through the delivery of light to light-sensitive microbial opsins, expressed in a genetically-defined population of cells (Cho et al., 2014). Opsins are seven-transmembrane proteins containing retinal, a vitamin A derived cofactor which isomerizes from its 11-cis configuration to an all-trans state upon photon absorption. This reaction triggers conformational changes in the opsin, resulting in ion flux. Notably, when expressed in mammalian tissue, opsins function as single-component systems that do not require exogenous retinal because this molecule is present in sufficient quantities in all vertebrate tissues tested to date (Fenno et al., 2011). Figure 1 summarizes the basic components of optogenetic systems. Although a variety of microbial opsins and mutant derivatives are available, a comprehensive discussion of the optogenetic 'toolbox' is beyond the scope of this review. Therefore, the opsins and chimera most relevant to examining cognition in schizophrenia are considered here.

A variety of opsins enabling neuronal excitation, altered excitability, and inhibition have been used in schizophrenia research. Excitation is commonly achieved using channelrhodopsin-2 (ChR2), as this channel depolarizes cells through the passive efflux of $\mathrm{K}^{+}$and influx of $\mathrm{Na}^{+}, \mathrm{Ca}^{2+}$, and $\mathrm{H}^{+}$in response to blue light (Lin, 2011). From $\mathrm{ChR}_{2}$, a number of chimera with altered activation/inactivation kinetics, desensitization properties, and temporal dynamics have been created (Fenno et al., 2011). Of these mutant proteins, the step function opsins (SFOs) have been used to examine cognition in schizophrenia. In particular, stabilized step function opsins (SSFOs) feature deactivation time constants nearing $30 \mathrm{~min}$, enabling prolonged depolarization and the study of chronic hyperexcitability in schizophrenia (Yizhar et al., 2011). Importantly, temporal resolution is not lost, as SSFOs/SFOs are rapidly activated and inactivated by blue and yellow light, respectively. Furthermore, ion-pumping microbial opsins, such as archaerhodopsin and halorhodopsin, impart hyperpolarization and therefore 'loss of function.' This is achieved via proton efflux in response to yellow or green light in archaerhodopsin (Arch) (Chow et al., 2012), or $\mathrm{Cl}^{-}$ influx in response to yellow light in halorhodopsin (NpHR) and enhanced halorhodopsin (eNpHR) (Fenno et al., 2011), 
an NpHR derivative with improved membrane targeting and higher photocurrents (Gradinaru et al., 2008).

Relative to ion channels, these pumps are limited by their ability to transport only a single ion across the cell membrane per photon, instead of allowing the simultaneous passage of numerous ions. Nonetheless, experiments using Arch, NpHR, and eNpHR are featured in this review, as they were the only available inhibitory optogenetic tools prior to the development of Step-waveform inhibitor channelrhodopsins (SwiChR). These channels were engineered through the introduction of specific mutations in the codon sequence of $\mathrm{ChR}$ to yield a $\mathrm{Cl}^{-}$conducting variant, which is rapidly activated and inactivated by blue and red light, respectively. Due to its rapid on/off kinetics and ability to conduct more ions per photon, SwiChR provides more physiologic inhibition of neurons (Berndt et al., 2014).

Prior to the development of optogenetics, researchers could not manipulate cell activity with the concurrent temporal and cellular specificity necessary for directly implicating PV interneurons in cognitive dysfunction. Transgenic organisms, pharmaceutical agents, and electrical stimulation were instead employed to alter neural activity. Although specific to cells with a certain expression profile, the pharmacological and genetic approaches lacked temporal resolution on the timescale of neural signaling. Electrical stimulation obviates this issue but does not enable cellular inactivation or specificity. Contrastingly, optogenetics attains cell-specificity by stereotactically injecting viruses encoding opsins, which are expressed under the control of a specific promoter, into the neuronal subpopulations under investigation (Fenno et al., 2011). In vivo, laser light is commonly delivered via a fiber optic cable implanted over the cells of interest, providing further cellular control (Huang et al., 2013). Additionally, a number of Cre-driver rodent lines and Cre-dependent viruses have been developed. In this system, Cre-recombinase, an enzyme required for the expression of Cre-dependent viruses, is restricted to the cell-type of interest, ensuring opsins are incorporated exclusively into such cells. Within these viruses, the opsin gene is present in an unreadable, inverted orientation, which is reversed by Cre-recombinase. The present discussion will feature experiments employing PV-Cre mice, which enable opsin expression at GABAergic PV interneurons. Temporal resolution, on the other hand, is derived from the millisecond activation/inactivation kinetics of microbial opsins, which impart optogenetic manipulations with a time scale comparable to physiological spiking (Fenno et al., 2011). Ultimately, optogenetics' combination of temporal and cellular precision provides unprecedented control over PV interneuron activity, allowing researchers to study these cells' contribution to the cognitive symptoms of schizophrenia as never before.

\section{PV interneuron dysfunction and gamma oscillations}

Optogenetics was initially applied to this area of research to disentangle whether PV interneurons governing oscillations in neural activity are associated with various types of cognition. Brain regions communicate through the synchronous firing of neuronal populations, represented as oscillatory extracellular field potentials on electroencephalography (EEG) recordings. These oscillations are classified on the basis of their frequency, typically as delta $(0-4 \mathrm{~Hz})$, alpha $(8-12 \mathrm{~Hz})$, beta $(12-30 \mathrm{~Hz})$, and gamma (30-200 Hz) (Williams et al., 2010). Decreased gamma-band activity is thought to underlie cognitive impairment in schizophrenia, as these oscillations are evoked in healthy individuals by a variety of perceptual and cognitive assessments (Cho et al. 2006). Consistent with this hypothesis, schizophrenia patients demonstrate reduced evoked gamma power on tasks measuring perceptual processing, executive control, and working memory (Uhlhaas and Singer, 2013). Electrophysiological studies indicate that these oscillations are the product of periodic fluctuations in pyramidal cell activity, driven by the rhythmic firing of cortical inhibitory interneurons which innervate these cells perisomatically (Lewis et al., 2012). Therefore, deficient GABAergic neurotransmission has been proposed to reduce the amplitude, or power, of cortical gamma oscillations, thereby contributing to the cognitive symptoms of schizophrenia. Indeed, reduced gamma power has been reported in both medicated and unmedicated patients with schizophrenia, suggesting it is likely an endophenotype of the disorder, rather than an iatrogenic effect (Williams et al., 2010).

Converging evidence from biochemical, pharmacological, and electrophysiological studies suggests reduced GABAergic transmission, particularly from PV interneurons, subserves the reduction in evoked gamma power characteristic of schizophrenia. Widely replicated post-mortem studies demonstrate alteration of GABAergic neurotransmission in this disorder. Relative to health controls, messenger RNAs (mRNA) of glutamic acid decarboxylase $\left(G A D_{67}\right)$, an enzyme involved in GABA synthesis, and the GABA membrane transporter 1 (GAT1) are decreased in cortical inhibitory interneurons of schizophrenia patients, suggesting reductions in GABA synthesis, reuptake, and transmission. PV interneurons appear to be particularly affected by these disturbances, as $\mathrm{GAD}_{67}$ mRNA is undetectable in $50 \%$ of these cells in brain samples from patients with schizophrenia. Moreover, data from several animal models of this disorder link decreased PV interneuron density to reduced gamma power (Uhlhaas and Singer, 2010). For example, rats treated with methylazoxymethanol acetate (MAM), a neurotoxin, demonstrate reduced PV interneuron densities in the medial 
prefrontal cortex (MPFC) and ventral subiculum. Local field potential recordings (LFPs) from MAM-treated rats reveal reduced evoked gamma oscillations in both regions during a test of latent inhibition, a type of associational learning commonly impaired in patients with schizophrenia (Lodge et al., 2009; McLaren et al., 2000). Nevertheless, these studies provide only correlative evidence linking PV interneuron dysfunction to reduced gamma power in schizophrenia, whereas optogenetic experiments have yielded causative evidence in support of this association.

Optogenetics has provided the first direct evidence indicating cortical PV interneurons drive gamma oscillations. To test whether PV interneurons contribute to the generation of gamma oscillations in vivo, Sohal and colleagues stereotactically injected a Cre-dependent Adenoassociated virus (AAV) encoding the eNpHR gene, under the control of the EF1 < promoter, into the prefrontal cortex (PFC) of PV-Cre mice. Anesthetized local field potential (LFP) recordings indicate stimulating PV interneurons with yellow light (593 $\mathrm{nm}$ ) exclusively reduces gamma power, suggesting these cells are critical to the normal production of only these oscillations (Sohal et al., 2009). Consistent with this finding, another group demonstrated that gamma oscillations are driven by rhythmic depolarizations at PV interneurons. To achieve this, a Cre-dependent AAV encoding an inverted $\mathrm{ChR}_{2}$ gene, controlled by the $\mathrm{EF}_{1}<$ promoter, was injected into the barrel cortex of PV-Cre mice. In contrast to the eNpHR experiment, the power of LFP recordings in anesthetized mice expressing $\mathrm{ChR}_{2}$ at $\mathrm{PV}$ interneurons was significantly amplified exclusively when the frequency of blue light $(473 \mathrm{~nm})$ stimulation corresponded to that most associated with evoked gamma oscillations in humans $(40 \mathrm{~Hz}$ ) (Cardin et al., 2009; TallonBaudry and Bertrand 1999). Taken together, these experiments provide the first direct evidence indicating cortical PV interneuron firing has a resonant, or natural, frequency in the gamma range. This suggests that the reduction in PV interneuron activity associated with schizophrenia should have deleterious effects on gamma oscillations, and therefore cognition.

Optogenetics has also implicated these cells, and the gamma oscillations they generate, in attention and sensory processing, cognitive functions frequently impaired in schizophrenia patients. Impaired attention has been documented in patients with schizophrenia, their firstdegree relatives, and those with schizophrenia-like personality disorders, suggesting it is an endophenotype of the disorder as well as a marker of increased genetic susceptibility (Chen et al., 2000). To examine the link between PV interneuron dysfunction and attention, researchers used an AAV to achieve expression of SwiChR channels in the medial prefrontal cortex (mPFC) of PV-Cre mice. Mice were then subjected to an attentional task, the three-choice serial reaction time task (3-CSRTT). Inhibition of PV interneurons with blue light $(473 \mathrm{~nm})$ resulted in a doubling of the total number of attentional errors, regardless of pulse duration ( $0.5,1.0$, or 2.0 seconds). In contrast, these researchers found expression of ChR in PV-Cre mice and subsequent stimulation of $\mathrm{PV}$ interneurons with blue light $(473 \mathrm{~nm})$ at frequencies within the gamma range $(30-40 \mathrm{~Hz})$ significantly decreased errors on the 3-CSRTT, relative to controls. These experiments provide direct evidence linking PV interneurons and the gamma oscillations they produce to attention, thereby suggesting the PV interneuron abnormalities documented in individuals with schizophrenia subserve the attentional deficits associated with the disease (Kim et al., 2016).

In addition to impairments in attention, individuals with schizophrenia demonstrate significant deficits in several types of cognition related to sensory processing, including sensory gating and multisensory integration (MSI) (Edgar et al., 2003). In particular, sensory gating describes the central nervous system's ability to suppress responses to irrelevant or redundant sensory inputs (Bourtros et al., 2004), whereas MSI is characterized by the binding of unisensory information to form a unified, multisensory precept (Stein and Stanford, 2008). The neural substrates of such sensory processes are not fully understood; however, cortical PV interneurons are thought to be involved. The cyclical inhibition these cells control is hypothesized to aid in cortical information processing by synchronizing the output of pyramidal cells on downstream neuronal assemblies participating in a common function, such as sensory encoding. More specifically, peak inhibition is thought to attenuate pyramidal cell responses to incoming sensory inputs, whereas a larger response should occur at the opposite portion of the gamma cycle (Cardin et al., 2009). Although pharmaceuticals known to disrupt gamma activity also impair rats on measures of sensory gating (Jones et al., 2014) and MSI (Jacklin et al., 2012), the effects of such drugs are not specific to PV interneurons. Therefore, these cells and gamma oscillations were not directly linked to sensory processing until the advent of optogenetics.

In one experiment, expression of $\mathrm{ChR}_{2}$ was achieved in barrel cortex PV interneurons of PV-Cre mice by injecting a Cre-dependent AAV, containing an inverted $\mathrm{ChR}_{2}$ gene and the EF1 < promoter, into this region. Gamma oscillations were induced in anesthetized mice by stimulating $\mathrm{ChR}_{2}$ with blue light $(473 \mathrm{~nm})$ at $40 \mathrm{~Hz}$. The effects of these oscillations on the transmission of ascending sensory information were examined by recording the response of pyramidal cells within the barrel cortex, following a single vibrissae deflection. As predicted, pyramidal cell responses were smaller when the rhythmic inhibitory post-synaptic potential (IPSP) produced by PV interneurons was at its peak. As the IPSP decayed, pyramidal cell responses increased in magnitude, demonstrating PV interneurons mediate sensory gating in a temporally specific manner (Cardin et al., 2009). These results suggest impaired 
cortical PV interneuron function and the resulting aberrations in gamma oscillations subserve the deficits in sensory processing often observed in patients with schizophrenia.

More recent optogenetic experiments have broadened our understanding of the cells thought to underlie cognitive dysfunction in this disorder to include PV interneurons within the basal forebrain (BF). While these cells have not yet been examined in post-mortem analyses of brain tissue from patients with schizophrenia, anatomical studies indicate they are ideally situated for controlling cortical PV interneurons. Specifically, BF PV interneurons project directly to their cortical counterparts, suggesting they regulate gamma activity and thus cognition (Kim et al., 2015). Because BF PV interneurons contribute to the sleepwake cycle (Xu et al., 2015), they are thought to control fluctuations in gamma oscillations associated with sleep, wakefulness, coma, and anesthesia (Vanderwolf, 2000). However, prior to the development of optogenetics, evidence for this hypothesis was sparse and derived largely from lesioning experiments in rodents. These indicate that cortically-projecting BF neurons regulate gamma activity (Kim et al., 2015) but lack the cellular precision necessary for defining the contribution of BF PV interneurons.

Optogenetic manipulations of these subcortical neurons' activity are more targeted, indicating that they control PV interneurons. To determine if excitation of BF PV interneurons alters cortical gamma oscillations, PV-Cre mice received unilateral $B F$ injections of a Cre-dependent AAV encoding an inverted $\mathrm{ChR}_{2}$ gene, controlled by the $\mathrm{EF}_{1}<$ promoter. Stimulation of BF PV interneurons with blue light $(473 \mathrm{~nm})$ at various frequencies $(2,10,20,30,40,50$, and 60 $\mathrm{Hz}$ ) revealed increased EEG power preferentially at $40 \mathrm{~Hz}$, suggesting these BF cells impart cortical PV interneurons with a frequency in the gamma range. Furthermore, stimulation of BF PV interneurons at frequencies reflecting their natural firing during REM sleep and wakefulness (20-60 $\mathrm{Hz}$ ) also enhanced cortical power exclusively at $40 \mathrm{~Hz}$. Taken together, these experiments demonstrate BF PV interneurons exert control over gamma oscillations in a state-dependent fashion. Although additional research is required to characterize these neurons' role in schizophrenia, this study is the first to suggest therapies modulating BF PV interneuron activity may prove particularly fruitful in restoring normal gamma oscillations and therefore cognition in individuals with this disorder (Kim et al., 2015).

\section{NMDA hypofunction: the molecular bases of PV interneuron dysfunction}

The molecular underpinnings of PV interneuron dysfunction have been examined using optogenetics as well. Specifically, hypofunctional N-methyl-D-aspartate receptors (NMDARs) are thought to underlie the reduction in GABAergic transmission from these cells in schizophrenia. This NMDAR hypofunction hypothesis purports that hypofunctional postsynaptic NMDARs lead to PV interneuron hypoexcitability. The resultant reduction in inhibitory outflow reduces cortical gamma power and contributes to cognitive deficits in schizophrenia (Cho and Sohal, 2014). This hypothesis is supported by studies demonstrating that subanesthetic doses of ketamine, an NMDAR antagonist, reproduce the positive, negative, and cognitive symptoms of the disorder in healthy individuals (Krystal et al., 2005). Similar to patients with schizophrenia, ketamine-treated rats exhibit reduced cortical gamma power (McNally et al., 2013) as well as impairments on tasks measuring sensory gating, latent inhibition (Becker et al., 2003), and multisensory integration (Jacklin et al., 2012). Moreover, anatomical studies suggest PV interneurons are especially sensitive to NMDAR hypofunction, as these cells receive a larger complement of glutamatergic afferents than interneurons lacking PV. In line with these data, long-term exposure to phencyclidine, an NMDAR antagonist, reduces PV mRNA in the rat PFC by $25 \%$ without altering the number of $\mathrm{PV}$ cells, suggesting NMDARs at these cells receive a high-degree of tonic glutamatergic activation (Cochran et al., 2003). Although these experiments link NMDA hypofunction to disturbances in PV interneurons and cognitive dysfunction, they lack the specificity necessary for establishing a causative relationship. Contrastingly, by providing both temporal and cellular precision, optogenetics has yielded the first direct evidence suggesting hypofunctional NMDARs, particularly on PV interneurons, are responsible for cognitive impairment in schizophrenia.

To date, optogenetics has been employed in a single study to examine the relationship between cognitive impairment and NMDA hypofunction at PV interneurons. For this experiment, a strain of PV-Cre mice expressing defective NMDARs on PV interneurons (PV-Cre/NR1f/f mice) received a bilateral barrel cortex injection of a Credependent AAV encoding ChR2. Subsequently, anesthetized electrophysiology was conducted while PV interneurons were stimulated with blue light $(473 \mathrm{~nm})$ at pulse frequencies corresponding to alpha $(8 \mathrm{~Hz}$ ), beta (24 $\mathrm{Hz})$, and gamma $(40 \mathrm{~Hz})$ oscillations. Relative to controls, $\mathrm{PV}$-Cre/NR1f/f mice exhibited a significant reduction in cortical power exclusively at $40 \mathrm{~Hz}$. These mice also demonstrated deficits in working memory, habituation, and associative learning but not anxiety. This finding suggests hypofunctional NMDARs on PV interneurons mediate the cognitive, rather than psychiatric, comorbidities of schizophrenia (Cosoff et al., 1998). Notably, these data are attributable to the loss of NMDAR signaling as immunohistochemistry confirmed normal cortical development as well as migration, and differentiation of PV interneurons from PV-Cre/NRıf/f mice. Unlike previously 
employed methods, this study reveals a causal relationship between hypofunctional NMDARs on PV interneurons, reduced gamma power, and cognitive impairments similar to those observed in schizophrenia patients. Despite the need for additional research to substantiate this link, this study suggests NMDA hypofunction as the principle pathology underlying impairments in PV interneuron function and cognition in schizophrenia (Carlén et al. 2012).

\section{PV interneurons in E/I imbalance}

Optogenetics has been employed to study the ramifications of NMDAR hypofunction at PV interneurons, predicted by the excitatory/inhibitory (E/I) imbalance hypothesis. This hypothesis posits that an elevated ratio of cortical cellular excitation to inhibition, due to increased excitatory neuron activity or impaired inhibitory neuron function, gives rise to the social and cognitive impairments seen in schizophrenia (Yizhar et al. 2011). Because of the large body of evidence demonstrating impaired PV interneuron function as a hallmark of this disorder, decreased inhibitory transmission is thought to drive E/l elevation. More specifically, this imbalance is attributed to NMDA hypofunction, which results in a reduction in GABAergic transmission, particularly at PV interneurons. The ensuing disinhibition of pyramidal cells is hypothesized to lead to the overstimulation of downstream excitatory neurotransmitter systems, accounting for imaging studies reporting elevated cortical glutamate in individuals with untreated schizophrenia (Poels et al., 2014).

Although the $\mathrm{E} / \mathrm{l}$ imbalance hypothesis eloquently unites several streams of pathophysiological evidence to predict the consequences of NMDA hypofunction, testing it has proven difficult. Due to a lack of pharmacological blockers specific to PV interneurons, the study of E/I imbalance has instead relied upon a combination of electrophysiology, transgenic animal models, and behavioural testing (Yizhar, 2012). In one experiment, the essential NR1 subunit of the NMDAR was ablated from 40$50 \%$ of hippocampal and cortical interneurons of mice in early postnatal development. During adolescence, the NR1 knockouts demonstrated social and cognitive impairments reminiscent of schizophrenia, in addition to reduced GAD67 and PV expression, also seen in post-mortem studies of schizophrenic brains. Additionally, in vivo tetrode recordings revealed increased pyramidal neuron firing rates, suggestive of a reduction in GABAergic outflow from inhibitory interneurons (Belforte et al., 2010). Despite upholding the behavioural and electrophysiological predictions made by the $E / /$ imbalance hypothesis, these results do not point to the cells underlying social and cognitive dysfunction in schizophrenia, as NR1 deletion was not specific to a particular type of interneuron. Due to a similar lack of cell specificity, experiments inducing E/l imbalance through cortical electrical stimulation have also failed to elucidate the neuronal subtype underlying this dysfunction (Yizhar, 2012).

In contrast to previous methods, optogenetics' unparalleled temporal and cellular control makes it ideally suited for elucidating the cellular substrates of E/l imbalance in schizophrenia. Toader et al. employed optogenetics and electrophysiology in combination to provide direct evidence in support of the cellular underpinnings of the $E / l$ imbalance hypothesis. In this experiment, an AAV-encoded inhibitory proton pump (Arch) was expressed in $\mathrm{PV}$ interneurons within the medial prefrontal cortex (mPFC) of PV-Cre mice. In addition to optic fibers, tetrodes were implanted bilaterally to record cellular activity. Upon light-induced inhibition of PV interneurons (525 nm), EEG recordings demonstrated decreased PV interneuron firing and increased firing of pyramidal neurons, a pattern consistent with the cellular mechanism of excess excitatory transmission purposed by the E/l imbalance hypothesis (Toader et al., 2019). Additional research has linked E/I imbalance at the cellular level to alterations in cognition and social behaviour similar to those seen in patients with schizophrenia. To modulate E/I balance, Yiszhar et al. expressed a stabilized SFO (SSFO) in both excitatory and inhibitory PV interneurons. In one group of mice, SSFO was expressed in excitatory PV cells by injecting an AAV encoding the SSFO gene, under the control of the CaMKII < promoter (CaMKII 〈:: SSFO), into the prelimbic and infralimbic regions of the mPFC. Contrastingly, SSFO expression in inhibitory PV cells was attained by injecting a Cre-dependent AAV containing an inverted SSFO gene, controlled by the PV (PV:: SSFO) promoter, into the same mPFC regions in PV-Cre mice. Elevated E/l balance was induced by stimulating PV cells in CaMKII 〈:: SSFO mice with blue light $(473 \mathrm{~nm})$, whereas the opposite was effected using the same protocol in PV::SSFO mice (Yizhar et al., 2011). Following SSFO stimulation, both groups were subjected to a behavioural battery. Similar to schizophrenia patients, the CaMKII 〈:: SSFO group demonstrated significant impairments on tasks measuring social novelty, episodic learning, and sociability. Although these data are consistent with the E/l imbalance hypothesis, this experiment did not incorporate NMDA hypofunction and used excitation rather than reduced inhibition to elevate E/l. As a result, future optogenetic experiments employing NMDAR deficient mice and inactivation of inhibitory PV interneurons via NpHR may be useful in more accurately characterizing the role these cells play in E/l imbalance. Despite these shortcomings, this study was the first to demonstrate that elevated mPFC E/l, mediated by PV interneurons, markedly disrupts conditioning and social behaviour in a manner comparable to schizophrenia (Yizhar et al., 2011). 


\section{Limitations and Future Directions}

The conclusions drawn from the aforementioned studies of PV interneurons are ultimately confined by optogenetics' finite cellular specificity, limited translatability, and inadequately characterized long-term effects. The majority of these experiments employed PV-Cre mice to restrict opsin expression to GABAergic PV interneurons. However, this technique does not yet permit optogenetic manipulations of PV interneuron subtypes due to a lack of viral vectors and promoters with sufficient specificity (Roux et al., 2014). Whether multiple or a single type of such cells contributes to the cognitive symptoms of schizophrenia remains unclear, as a result. Additionally, optogenetics has not received approval for use in humans with schizophrenia. The translatability of the studies presented here is therefore hindered by the reliance on rodents, organisms which lack the cognitive and social complexity of humans (Yizhar, 2012). The paucity of optogenetic experiments examining cognition in well-established animal models of schizophrenia compounds this issue of translatability further. Such models will likely provide more robust evidence demonstrating dysfunctional PV interneurons as the neural correlates of cognitive impairment associated with the disorder. Lastly, the long-term tolerability of microbial opsins and light stimulation have not been adequately studied (Fenno et al., 2011). Characterizing these factors ensures data are attributable to optogenetic alterations of cell-activity, rather than immune reactions to microbial opsins or thermally-induced neuronal damage, resulting from repeated optical stimulation.

Despite these constraints, the impact of optogenetics on the study of cognitive dysfunction in schizophrenia suggests that it will likely become a mainstay in the 'toolbox' of researchers in this field. With an increasing number of opsins available, the possible future directions are seemingly limitless. However, studies employing opto-XRs, optically-sensitive G-protein coupled receptors (GPCRs) linked to intracellular signaling pathways, may prove particularly fruitful in furthering our understanding of cognitive impairment and identifying new molecular targets for pharmaceutical development. To date, optogenetics has been used to study the cognitive comorbidities of schizophrenia exclusively at the cellular and circuit-levels. Such research is critical to our understanding of cognition in both physiological and pathophysiological states but does not define abnormal intracellular pathways, relevant to the development of cognition-enhancing pharmaceuticals. Opto-XRs have the potential to resolve this issue, as they enable targeted activation of $G_{s}$ and $G_{q}$ signaling (Bernstein and Boyden, 2011; Fenno et al., 2011). To highlight the utility of these optogenetic tools in uncovering the molecular bases of PV interneuron dysfunction, a potential future experiment is outlined below.
Using opto-XRs, we can probe how novel antipsychotics, such as phosphodiesterase inhibitors (PDEIs), ameliorate PV interneuron function and cognition in schizophrenia patients. PDEls block phosphodiesterase, an enzyme critical to the breakdown of the second messenger cyclic adenosine monophosphate (cAMP) (Raheem et al., 2015). The resulting increase in intracellular CAMP is hypothesized to activate proteins experiencing low levels of activation due to NMDAR hypofunction. Specifically, the cognition-enhancing effects of these drugs may result from an increase in CAMP response element-binding protein (CREB) activation. Under physiological conditions, NMDARs enable $\mathrm{Ca}^{2+}$ influx which stimulates two parallel intracellular pathways, both culminating in the activation of CREB, a transcription factor. Importantly, CAMP activates this protein independent of the NMDAR pathway (Hardingham and Bading, 2003). It can be determined whether the cognitive effects of PDEls result from increased cAMP using an opto-XR linked to $G_{s,}$ a $G$-protein which stimulates the production of cAMP by activating adenylyl cyclase (Morello et al., 2009). Expressing these opto-XRs in transgenic mice with hypofunctional NMDARs on PV interneurons (PVCre/NR1f/f mice) allows us to examine how PDEls bypass this dysfunction. Following optical stimulation, rescue from the learning and memory deficits characteristic of these mice (Carlén et al. 2012) would suggest PDEls mediate cognitive enhancement through an increase in intracellular CAMP. Such results could lead to the use of already available PDEls in the treatment of schizophrenia and direct the synthesis of novel, cAMP-enhancing antipsychotics.

\section{Conclusion}

Optogenetics has provided an unparalleled glimpse into the cellular mechanisms contributing to cognitive dysfunction in schizophrenia, suggesting the technique has a bright future in schizophrenia research. In contrast to previously employed methods, optogenetic manipulations of cell activity are both spatially and temporally specific, enabling unprecedented control of neural circuits and their constituent cells. Thus, this technique is uniquely suited for pinpointing how dysfunctional PV interneurons contribute to the cognitive symptoms of schizophrenia. Optogenetics' applicability to this area of research is particularly reflected in the direct associations it has established between multiple, once tangentially-related lines of evidence. Specifically, experiments using this tool are the first to reveal causal relationships between cortical and subcortical PV interneurons and the generation of gamma oscillations associated with various cognitive processes often impaired in schizophrenia. Such studies also link both hypofunctional NMDARs and E/l imbalance at PV cells to aberrations in gamma oscillations and behaviour consistent with this disorder. By more concretely connecting existing 
hypotheses, optogenetics has provided us with a more integrated understanding of the cognitive deficits of schizophrenia. Ultimately, this newly expanded perspective identifies PV interneurons and the signaling cascades within as targets for the development of novel antipsychotics, while advancing our comprehension of arguably the most scientifically perplexing illnesses affecting humanity.

\section{Acknowledgements}

I would like to thank Dr. John Howland for his guidance on this work. 


\section{References}

Addington J (1991). Cognitive functioning and positive and negative symptoms in schizophrenia.

Schizophrenia Research 5:123-34.

Belforte JE, Zsiros V, Sklar ER, Jiang Z, Yu G, Li Y, Quinlan EM, Nakazawa K (2010). Postnatal NMDA receptor ablation in corticolimbic interneurons confers schizophrenia-like phenotypes. Nature Neuroscience 13: 76-83.

Berndt A, Lee SY, Ramakrishnan C, Deisseroth K (2014). Structure-guided transformation of channelrhodopsin into a light-activated chloride channel. Science 344: 420-424.

Bernstein JG, Boyden ES (2011). Optogenetic tools for analyzing the neural circuits of behavior. Trends in Cognitive Science 15: 592-600.

Boutros NN, Korzyukov O, Jansen B, Feingold A, Bell M (2004). Sensory gating deficits during the midlatency phase of information processing in medicated schizophrenia patients. Psychiatry Research 26: 203-15.

Cardin JA, Carle M, Meletis K, Knoblich U, Zhang F, Deisseroth K, Li-Huei T, Moore Cl (2009). Driving fast-spiking cells induces gamma rhythm and controls sensory responses. Nature 459: 663-8.

Carlen M, Meletis K, Siegle JH, Cardin JA, Futai K, Ruhlmann C, Jones SR, Deisseroth $\mathrm{K}$, Sheng $\mathrm{M}$, Moore Ci, Tsai L-H (2012). A critical role for NMDA receptors in parvalbumin interneurons for gamma rhythm induction and behavior. Molecular Psychiatry 17: 537-48.

Chen WJ, Faraone S. Sustained attention deficits as markers of genetic susceptibility to schizophrenia (2000). American Journal of Medical Genetics 97: 5257.

Cho RY, Konecky RO, Carter CS (2006). Impairments in frontal cortical gamma synchrony and cognitive control in schizophrenia. Proceedings of the National Academy of Science 103:19878-83.

Cho KKA, Sohal VS (2014). Optogenetic approaches for investigating neural pathways implicated in schizophrenia and related disorders. Human Molecular Genetics 23:64-8.
Chow BY, Han X, Boyden ES (2012). Genetically encoded molecular tools for light-driven silencing of targeted neurons. Progress in Brain Research 196: 49-61.

Claudia PEA, Lorenza C, Bernhard H (2013). File: optogenetic stimulation consists of several steps. Wiki commons. Retrived from: https://commons.wikimedia.org/wiki/File:Optogen etic_stimulation_consists_of_several_steps.png

Cochran SM, Kennedy M, Mckerchar CE, Steward LJ, Pratt JA, Morris BJ (2003). Induction of Metabolic Hypofunction and Neurochemical Deficits after Chronic Intermittent Exposure to Phencyclidine : Differential Modulation by Antipsychotic Drugs. Neuropsychopharmacology 28: 265-75.

Cosoff SJ, Hafner RJ (1998). The prevalence of comorbid anxiety in schizophrenia, schizoaffective disorder and bipolar disorder. Australian and New Zealand Journal of Psychiatry 32: 67-72.

Cunningham MO, Hunt J, Middleton S, Lebeau FEN, Gillies MG, Davies CH (2006). Region-Specific Reduction in Entorhinal Gamma Oscillations and Parvalbumin-Immunoreactive Neurons in Animal Models of Psychiatric Illness. The Journal of Neuroscience 26: 2767-76.

Edgar CJ, Moses S, Thoma RJ, Huang M, Hanlon F, Weisend MP, Bustillo J, Miller GA, Adler LE, Canive JM (2003). Cross-model generality of the gating deficit in schizophrenia. Psychophysiology 42: 318-327.

Fenno L, Yizhar O, Deisseroth K (2011). The Development and Application of Optogenetics. Annual Review of Neuroscience 34: 389-412.

Geyer M, Gross G. Novel Antischizophrenia Treatments. Springer-Verlag, 2012.

Gradinaru V, Thompson KR, Deisseroth K (2008). eNpHR: a Natronomonas halorhodopsin enhanced for optogenetic applications. Brain Cell Biology 36:129-39.

Hardingham GE (2003). Bading H. The Yin and Yang of NMDA receptor signalling. Trends in Neuroscience 26: 81-9.

Huang F, Tang B, Jiang H (2013). Optogenetic investigation of neuropsychiatric diseases. International Journal of Neuroscience 123: 7-16. 
Jacklin DL, Goel Amit, Clementino KJ, Hall AWM, Talpos JC, Winters BD (2012). Severe cross-modal object recognition deficits in rats treated sub-chronically with NMDA receptor antagonists are reversed by systemic nicotine: implications for abnormal multisensory integration in schizophrenia.

Neuropsychopharmacology 37: 2322-2331.

Jones NC, Anderson P, Rind G, Sullivan C, Buuse M Van Den, Brien TJO (2014). Effects of aberrant gamma frequency oscillations on prepulse inhibition. International Journal of Neuropsychopharmacology 17: 1671-81.

Kim H, Ahrlund-Richter S, Wang Z, Deisseroth K, Carlen M. Prefrontal parvalbumin neurons in control of attention. Cell 164: 208-218.

Kim T, Thankachan S, Mckenna JT, Mcnally JM, Yang C, Choi JH, Chen L, Kocsis B, Deisseroth K, Strecker RE, Basheer R, Brown RE, McCarley Rw (2015). Cortically projecting basal forebrain parvalbumin neurons regulate cortical gamma band oscillations. Proceeding of the National Academy of Science 112: 3535-3540.

Levkovitz Y, Mendlovich S, Riwkes S, Braw Y, Levkovitchverbin H, Gal G (2010). A Double-Blind, Randomized Study of Minocycline for the Treatment of Negative and Cognitive Symptoms in Early-Phase Schizophrenia. The Journal of Clinical Psychiatry 71:138-149.

Lewis DA, Lieberman JA (2000). Catching Up on Schizophrenia : Natural History and Neurobiology. Neuron 28:325-34.

Lewis DA, Curley AA, Glausier JR, Volk DW (2012). Cortical parvalbumin interneurons and cognitive dysfunction in schizophrenia. Trends in Neuroscience 35: 57-67.

Lin JY (2011). A user's guide to channelrhodopsin variants: features, limitations and future developments. Experimental Physiology 96: 19-25.

Lodge DJ, Behrens MM, Grace AA (2009). A Loss of Parvalbumin-Containing Interneurons Is Associated with Diminished Oscillatory Activity in an Animal Model of Schizophrenia. Neurobiology of Disease 29: 2344-54.

McLaren IPL, Mackintosh NJ (2000). An elemental model of associative learning: I. latent inhibition and perceptual learning. Animal Learning \& Behavior 28: $211-246$.

Morello S, Sorrentina R, Pinto A (2009). Adenosine A2a receptor agonists as regulators of inflammation : pharmacology and therapeutic opportunities. Journal of Receptor, Ligand and Channel Research 2: 11-7.

Poels EMP, Kegeles LS, Kantrowitz JT, Slifstein M, Javitt DC, Lieberman JA, Abi-Dargham A, Girgis RR (2013). Imaging glutamate in schizophrenia: review of findings and implications for drug discovery. Molecular Psychiatry 19: 20-9.

Raheem IT, Schreier JD, Fuerst J, Gantert L, Hostetler ED, Huszar S, Joshi A, Kandebp M, Kim SH, Li J, Ma B, McGaughey G, Sharma S, Shipe WD, Uslaner J, Vandeveer GH, Yan Y, Renger JJ, Smith SM, Coleman PJ, Cox CD (2016). Discovery of pyrazolopyrimidine phosphodiesterase $10 \mathrm{~A}$ inhibitors for the treatment of schizophrenia. Bioorganic Medicinal Chemistry Letter 26:126-32.

Roux L, Stark E, Sjulson L, Buzsaki G (2015). In vivo optogenetic identification and manipulation of GABAergic interneuron subtypes. Current Opinions in Neurobiology 0: 88-95.

Sohal VS, Zhang F, Yizhar O, Deisseroth K (2009). Parvalbumin neurons and gamma rhythms enhance cortical circuit performance. Nature 459: 698-702.

Stein B, Stanford T (2008). Multisensory integration: of the single neuron. Nature Neuroscience 9: 255-266.

Tallon-Baudry C, Bertrand O (1999). Oscillatory gamma activity in humans and its role in object representation. Trends in Cognitive Sciences 3: 151162.

Toader O, Nissen W, Schülert N, Von Heimendahl M, Rosenbrock H (2019). Effects of metabotropic glutamate $2 / 3$ receptor activation on deficits of neural network function induced by optogenetic inhibition of parvalbumin-positive interneurons in the mouse prefrontal cortex. Schizophrenia International Research Society: 315.

Uhlhaas PJ, Singer W(2010). Abnormal neural oscillations and synchrony in schizophrenia. Nature Reviews Neuroscience 11:100-13. 
Uhlhaas PJ, Singer W (2013). High frequency oscillations and the neurobiology of schizophrenia.

Translational Research 15: 301-13.

Vanderwolf $\mathrm{CH}$ (2000). Are neocortical gamma waves related to consciousness? Brain Research 855: 21724.

Williams S, Boksa P (2010). Gamma oscillations and schizophrenia. Journal of Psychiatry \& Neuroscience 35:75-7.

Yizhar O, Fenno LE, Prigge M, Schneider F, Davidson TJ, Shea DJO, Sohal VS, Goshen I, Finkelstein J, Paz JT, Stehfest K, Fudim R, Ramakrishnan C, Huguenard JR, Hegemann P, Deisseroth K (2011). Neocortical excitation / inhibition balance in information processing and social dysfunction. Nature 477: 171-8.

Yizhar O (2012). Optogenetic Insights into Social Behavior Function. Biological Psychiatry 71: 1075-80. 
Optogenetics Implicates PV Interneurones in Schiziphrenia (Parker)

University of Saskatchewan Undergraduate Research Journal 